\title{
ÍNDICES TÉCNICOS E RENTABILIDADE DA PECUÁRIA LEITEIRA ${ }^{1}$
}

\author{
Terezinha Bezerra Albino Oliveira ${ }^{2 *}$; Reginaldo Santana Figueiredo ${ }^{3}$; Mauro Wagner de Oliveira4; \\ Christiano Nascif 5 \\ ${ }^{2}$ Depto. de Administração-UFV, CEP: 36571-000 - Viçosa, MG. \\ ${ }^{3}$ Depto. de Engenharia de Produção-UFSCar, C.P. 676 - CEP: 13565-905 - São Carlos, SP. \\ ${ }^{4}$ Centro de Pesquisa e Melhoramento de Cana-de-Açúcar - UFV, C.P. 342 - CEP: 35430-970 - Ponte Nova, MG. \\ ${ }^{5}$ Convênio Nestlé/FUNARBE/UFV, CEP: 36571-000 - Viçosa, MG. \\ *Autor correspondente <tbalbino@ufv.br>
}

\begin{abstract}
RESUMO: Avaliaram-se os resultados técnicos e econômicos em 22 propriedades leiteiras localizadas em dez municípios da Região de Viçosa, MG. Nas propriedades que apresentaram resultados econômicos negativos, detectaram-se os pontos de estrangulamento na produção e, tomando-se como referência os índices técnicos das propriedades que apresentaram taxa de retorno sobre o investimento superior ao da caderneta de poupança, foram propostas medidas para aumentar a eficiência dessas fazendas. A escala de produção foi considerada fator importante para obtenção de índices econômicos atrativos. A melhoria do padrão genético, através de inseminação artificial com sêmen de animais com maior potencial produtivo, contribuiu para elevar os índices de produtividade do rebanho, da terra e da mão-de-obra e, consequentemente os indicadores econômicos. A alimentação adequada, principalmente das vacas em lactação, foi muito importante para o sucesso da atividade. Investimento em qualidade do leite via controle sanitário do rebanho, ordenha mecanizada e resfriamento logo após a ordenha, podem ser uma estratégia para obter ganhos em toda a cadeia produtiva.
\end{abstract}

Palavras-chave: análise técnica, análise econômica, escala de produção

\section{TECHNICAL INDICES AND PROFITABILITY OF THE DAIRY INDUSTRY}

\begin{abstract}
Economic and technical results of a study involving 22 dairy farms in 10 municipalities of Viçosa, MG, Brazil, were evaluated. In the properties that presented negative economic results, problems were detected in the production phase. Technical indexes of the properties that presented a return rate higher than that of savings investment were taken as reference, and measures were proposed to increase the efficiency of the other farms. The scale of production is of the utmost importance to enhance dairy farm interest. Genetic standard improvement by means of artificial insemination with semen of animals with high productive potential has contributed to raise the productivity indexes of the herd, land and labor and, consequently, the economic indexes. Food and animal environment were relevant factors for the activity success. Increased milk quality via mechanized milking immediately followed by cooling has generated gains throughout the whole productive process.
\end{abstract}

Key words: technical analysis, economic analysis, production scale

\section{INTRODUÇÃO}

As atividades agrícolas conduzidas com fins lucrativos devem ser contabilizadas para periódicas análises do desempenho econômico e técnico. Entretanto, poucas são as propriedades rurais de pequeno e médio portes que contabilizam suas atividades para posterior análise econômica, e, por isto, não conhecem seus custos de produção de leite, especialmente os custos fixos. Assim, a inexistência de fontes de informações confiáveis levam os produtores à tomada de decisão condicionada à sua experiência, à tradição, ao potencial da região, à falta de outras opções e à disponibilidade de recursos financeiros e de mão-de-obra. Quando a rentabilidade é baixa, o produtor percebe, mas tem dificuldade em quantificar e identificar os pontos de estrangulamento do processo produtivo. A incorporação do uso da informática à vida do homem do campo, com o desenvolvimento de novos softwares, veio colaborar para melhor planejamento e avaliação da atividade produtiva.

A lucratividade da pecuária leiteira depende do preço do leite, comparativamente aos preços dos insumos e fatores de produção (relação de troca) e também da quantidade produzida em relação às quantidades usadas dos fatores de produção (produtividade).

O pecuarista aumenta sua margem de lucro à medida que ele consegue incrementar o volume de produção que, além de ser importante na redução dos elevados custos fixos, é fator de vantagens diferenciais concedidas pela indústria compradora, principalmente no transporte e na qualidade da matéria-prima. Gomes (1997) demonstrou em pesquisa realizada em fazendas produtoras de leite no Estado de São Paulo que a escala de produção tem influência decisiva na maximização do lucro, visto que o produtor trabalha com pequena margem de lucro por litro de leite.

${ }^{1}$ Parte da Dissertação de Mestrado da primeira autora apresentada à UFSCar - São Carlos, SP. 
O desempenho técnico-econômico da atividade leiteira pode, ser avaliado através de vários índices técnicos, da relação entre eles e também pela análise econômica. Como índices técnicos têm-se utilizado, dentre outros: 1) produção média por vaca em lactação/ dia; 2) produção média diária pelo total de vacas do rebanho; 3) produção de leite por hectare/ano; 4) taxa de natalidade; 5) idade ao primeiro parto; 6) intervalo entre partos; 7) litros de leite por quilo de concentrado fornecido; e 8) mão-de-obra por litro de leite produzido (Martins, 1988; Federação da Agricultura do Estado do Paraná, 1996; Gomes, 1997; Schiffler, 1998). Na análise de desempenho econômico, os principais indicadores utilizados têm sido a margem bruta, a margem líquida e a taxa de retorno sobre o investimento.

O objetivo deste trabalho foi avaliar os índices técnicos e a rentabilidade da atividade leiteira de vinte e duas propriedades assistidas por um programa de desenvolvimento da pecuária leiteira da Região de Viçosa, Minas Gerais (PDPL-RV).

\section{MATERIAL E MÉTODOS}

Devido às deficiências existentes nas propriedades rurais, no que diz respeito à escrituração para o controle da atividade, os dados necessários para a operacionalização do estudo, coletados durante dois anos, entre maio de 1996 e abril de 1998, subdivididos em estação das secas (maio a outubro) e estação das águas (novembro a abril), foram obtidos junto ao Programa de Desenvolvimento da Pecuária Leiteira da Região de Viçosa, Minas Gerais (PDPL-RV), um convênio entre a Nestlé Brasil Ltda e a Universidade Federal de Viçosa-UFV, tendo como executora a Fundação Arthur Bernardes-Funarbe, órgão vinculado à UFV.

Assim, através de consulta à base de dados do PDPL-RV, e a partir dos valores médios dos quatro períodos, calculou-se o desempenho médio para:

\section{a) Indicadores de desempenho econômico:}

Margem Bruta (MB): em termos absolutos é a diferença entre a receita total e o custo operacional efetivo (COE). Também pode ser expressa em termos percentuais (MB\%), dividindo-se seu valor absoluto pela receita e multiplicando-se por 100 . É o montante que vai remunerar os fatores fixos da atividade tais como terra, animais, benfeitorias, máquinas e a mão-de-obra familiar. O COE compõem-se dos desembolsos efetivamente realizados na condução da atividade, exceto a mão-deobra familiar e a depreciação.

Margem Líquida (ML): em termos absolutos é a diferença entre receita total ou renda bruta e o custo operacional total (COT) ou, também, pode ser expressa em termos percentuais $\mathrm{ML}(\%)$, dividindo-se seu valor absoluto pela receita e multiplicando-se por 100 . Portanto, a ML(\%) indica a percentagem que restou de cada $R \$ 1,00$ de receita após a dedução de todos os custos. É o resíduo que remunera o capital investido na atividade leiteira. O COT é o custo de reposição do capital efetivamente empregado, uma vez que os investimentos já foram realizados. É o COE mais a mãode-obra familiar e a depreciação.

Taxa Mensal de Retorno Sobre o Investimento (\%): é o percentual resultante da divisão entre a ML e o capital investido sem ou com terra. Indica quanto a empresa ganha para cada $\mathrm{R} \$ 100,00$ de capital investido.

\section{b) Indicadores de desempenho técnico:}

Produção Diária (PD): definida pela quantidade média mensal de leite vendida ou autoconsumida na forma fluida ou na forma de derivados expressos na quantidade equivalente de litros de leite $\left(\mathrm{L} \mathrm{dia}^{-1}\right)$;

Relação Vacas em Lactação/Total de Vacas (RVL/TV): é o percentual de vacas em lactação em relação ao total de vacas (secas e em lactação) no rebanho;

Produção por Vaca em Lactação (PVL): é a relação entre a produção média de leite e número de vacas em lactação (L/vacas lactação/dia);

Produtividade da Terra (PT): é a relação entre a produção média diária e a área destinada à pecuária leiteira, expressa em litros por hectare ano;

\section{Produtividade da Mão-de-Obra Permanente} (PMO): é a relação entre a produção média diária e a mão-de-obra utilizada na exploração da atividade, medida em litros por dia trabalhado;

Concentrado por Litro de Leite (CLL): é a relação entre a quantidade de concentrado fornecida às vacas em lactação e a produção de leite obtida do período, medida em kg ração por litro de leite.

As análises técnicas e econômicas foram realizadas seguindo método descrito por Gomes et al. (1986); Embrapa (1991); Melo Filho \& Richetti (1998), enquanto os parâmetros indicadores de eficiência foram definidos com base nos autores acima citados e em Matarazzo (1992); Federação da Agricultura do Estado do Paraná (1996); Mondani (1996); Gomes (1997).

Após terem sido concluídas as análises econômicas e técnicas, realizou-se visitas técnicas às propriedades para se confirmar "in loco" a viabilidade das medidas propostas com vistas ao aumento da eficiência e da rentabilidade dessas fazendas.

\section{RESULTADOS E DISCUSSÃO}

O valor médio mensal dos indicadores econômicos e técnicos, obtidos no levantamento de dados dos quatro períodos, encontram-se nas TABELAS 1 e 2 . Pela análise da 1 , verifica-se que as propriedades de números 1 a 7,9 a 11 e 13 apresentaram valores 
médios positivos para os três indicadores econômicos analisados: margem bruta (MB), margem líquida (ML), taxa de retorno sobre o investimento considerando a terra (TRI). As propriedades de número 8, 14 e 17, por terem obtido pequena margem bruta positiva, $R \$ 3,65$; $\mathrm{R} \$ 64,08$; e $\mathrm{R} \$ 29,61$, respectivamente, passaram a ter valores negativos para os outros dois indicadores econômicos. A propriedade de número 18 , embora com $M B$ média de $R \$ 256,55$, também teve $M L$ e TRI negativas, devido ao alto valor de depreciação ponderada no período $(21,7 \%)$, utilizada nos cálculos.

Os maiores valores de MB, ML e TRI foram obtidos pelas propriedades número 1 e 2 . Para a propriedade número 1 a TRI de 1,2\% superou, à época, em quase o dobro o investimento em caderneta de poupança, considerado como referência de aplicação financeira (Martins, 1988; Gomes, 1997; Melo Filho \& Richetti, 1998).

A produção diária, a venda de animais, os índices técnicos de PVL, RVL/TV e a capacidade de gerenciamento dos outros fatores de produção contribuíram para o bom desempenho econômico destas duas propriedades (TABELA 2). A produção por vaca em lactação, o percentual de vacas em lactação e a quantidade de leite produzida (Federação da Agricultura do Estado de Minas Gerais, 1996; Schiffler, 1998), associados à receita proveniente da venda de animais (Federação da Agricultura do Estado do Paraná, 1996), têm sido apontados como fatores responsáveis pela maior rentabilidade da atividade leiteira.

Para as propriedades de número 3 a 7,9 a 11 e 13, embora a TRI tenha sido positiva, os resultados econômicos não foram atrativos, comparativamente aos da caderneta de poupança. Assim, há necessidade de maior eficiência nestas propriedades, para se conseguir aumento na produção diária, como uma das formas de reduzir os custos fixos por litro de leite, à semelhança do que foi proposto por Schiffler (1998), para sistema de produção de leite na Região de São Carlos, SP.

Ainda pela análise da TABELA 1, verifica-se que as propriedades de número 12, 15, 16 e 19 a 22 tiveram margem bruta negativa. Para margem líquida e retorno sobre investimento, além dessas propriedades, as de número $8,14,17$ e 18 também apresentaram resultados negativos para estes dois índices econômicos.

A melhoria dos índices técnicos destas propriedades poderá levar à melhoria dos resultados econômicos. Assim, foram tomados como referência os índices técnicos obtidos nas propriedades de número 1 e 2 (TABELA 2), agrupando-se as propriedades de número $8,12,14$ a 22, conforme aceitação ou não de seus índices técnicos. Estabeleceram-se índices mínimos a serem alcançados: RVL/TV de $70 \%$, produção diária de 100 litros, produção média por vacas em lactação de 10 litros/dia, produtividade da terra de $1.000 \mathrm{~L} \mathrm{ha}^{-1}$ ano $^{-1}$ e produtividade da mão-de-obra de $100 \mathrm{~L} \mathrm{~d}^{-1} \mathrm{~h}^{-1}$ e o valor máximo de $0,300 \mathrm{~kg}$ de concentrado por litro de leite produzido. Os valores dos índices técnicos que estão fora dos limites estipulados encontram-se sublinhados e em negrito na TABELA 3.

Uma vez que a escala de produção tem grande influência no lucro da atividade leiteira, há necessidade de se propor alternativas para aumento de produção diária de leite. As alternativas para aumentar a produção seriam: a) aumento do número de vacas em lactação no rebanho; e b) aumento da produtividade animal (litros de leite/dia). Para a primeira alternativa, os fatores limitantes seriam, dentre outros, os recursos financeiros para aquisição de vacas e a disponibilidade de área para alimentação desses animais. Nas visitas técnicas, verificou-se que várias propriedades possuem taxa de lotação inferior a $1 \mathrm{UA} /$ ha e assim, teriam, nesta opção, uma forma de aumento de vacas em lactação no rebanho e, conseqüentemente, aumento da produção diária de leite com esses acréscimos na taxa de lotação. A segunda alternativa proposta para aumento de produção seria aumentar a produtividade animal. Várias têm sido as alternativas propostas para a melhoria da produtividade do rebanho. Essas alternativas incluem, dentre outros, aumento do percentual de vacas em lactação no rebanho (RVL/TV), melhoria no padrão

TABELA 1 - Média mensal dos indicadores econômicos, no período de dois anos, das 22 propriedades leiteiras analisadas.

\begin{tabular}{|c|c|c|c|}
\hline Propriedade & ${ }^{*} \mathrm{MB}$ & ${ }^{*} \mathrm{MB}$ & ${ }^{*} \mathrm{TRI}$ \\
\hline & \multicolumn{2}{|c|}{ 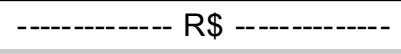 } & $\%$ \\
\hline 1 & $7.963,25$ & $7.674,20$ & 1,23 \\
\hline 2 & $4.094,66$ & $3.381,71$ & 0,70 \\
\hline 3 & $1.533,20$ & $1.220,77$ & 0,16 \\
\hline 4 & $1.035,20$ & 159,34 & 0,06 \\
\hline 5 & 687,24 & 511,48 & 0,10 \\
\hline 6 & 456,79 & 171,11 & 0,11 \\
\hline 7 & 709,56 & 411,08 & 0,26 \\
\hline 8 & 3,65 & $-139,33$ & $-0,06$ \\
\hline 9 & 202,82 & 169,10 & 0,20 \\
\hline 10 & 703,66 & 299,07 & 0,08 \\
\hline 11 & 395,78 & 88,80 & 0,03 \\
\hline 12 & $-214,39$ & $-508,30$ & $-0,29$ \\
\hline 13 & 451,95 & 101,58 & 0,05 \\
\hline 14 & 64,08 & $-28,77$ & $-0,03$ \\
\hline 15 & $-367,89$ & $-405,26$ & $-1,12$ \\
\hline 16 & $-178,35$ & $-282,40$ & $-0,30$ \\
\hline 17 & 29,61 & $-106,11$ & $-0,09$ \\
\hline 18 & 256,55 & $-7,08$ & $-0,01$ \\
\hline 19 & $-309,18$ & $-451,54$ & $-0,33$ \\
\hline 20 & $-381,29$ & $-718,08$ & $-0,17$ \\
\hline 21 & $-615,05$ & $-701,84$ & $-0,82$ \\
\hline 22 & $-47,96$ & $-198,76$ & $-0,17$ \\
\hline
\end{tabular}

${ }^{*} \mathrm{MB}=$ Margem bruta; $\mathrm{ML}=$ Margem líquida; $\mathrm{TRI}=$ Taxa de retorno sobre o investimento considerando a terra. 
genético do rebanho, na alimentação, na sanidade e na ambiência. O aumento do percentual de vacas em lactação no rebanho pode ser conseguido através da redução do intervalo entre partos.

A diminuição do intervalo entre partos é resultado da redução do início da nova gestação após o parto e do período de descanso. Para atingir esse objetivo deverá ser avaliada a alimentação dos animais, sua sanidade e também o padrão racial. $O$ ideal é se ter um intervalo entre partos de 12 meses, proporcionando uma cria por ano.

As propriedades de $n^{\circ} 7,8,12$ e 18 (TABELA 2) têm percentual de vacas em lactação próximo ou superior a $80 \%$. Considerando um período de gestação de nove meses e 20 dias, com início de nova gestação aos 70 dias após o parto e um período ideal de descanso de 60 dias, a relação de vacas em lactação será de aproximadamente $83 \%$. Segundo Faria (1988), uma propriedade ou um sistema de produção é considerado eficiente quando a vaca mostra persistência na produção de leite e se reproduz de forma regular, isto é, mantém o estabelecimento de prenhez próximo a 70 dias após o parto. Períodos de descanso inferiores a 50 dias não têm sido recomendados, devido ao comprometimento da próxima lactação, pois sabe-se que nas últimas 3-4 semanas há grande aumento nas exigências energéticas e protéicas do feto.

As demais propriedades (1 a 6, 9 a 11, 13 a $17 \mathrm{e}$ 19 a 22) poderão obter aumento de produtividade simplesmente elevando o percentual de vacas em lactação no rebanho; contudo, somente três propriedades possuem percentual de vacas em lactação no rebanho inferior a $60 \%$ (TABELA 2).

A característica racial é fator determinante da alta relação do percentual de vacas em lactação no rebanho, pois conhecidamente as raças européias, mais notadamente a holandesa, sob condições normais de alimentação e sanidade animal, apresentam intervalo entre partos em torno de 12 meses (Federação da Agricultura do Estado do Paraná, 1996). Para a Região da Zona da Mata Mineira, estudos conduzidos pela Federação da Agricultura do Estado de Minas Gerais (1996) constataram percentual de vacas em lactação oscilando em torno de $65 \%$ e, no Brasil, este percentual médio é de 50\% (Faria \& Silva, 1996). Certamente, maior

TABELA 2 - Média mensal dos indicadores técnicos, no período de dois anos, das 22 propriedades leiteiras analisadas*.

\begin{tabular}{|c|c|c|c|c|c|c|c|c|c|c|c|c|c|c|c|c|c|c|}
\hline \multirow{3}{*}{$\begin{array}{c}\mathrm{PR}^{* *} \\
1\end{array}$} & \multirow{2}{*}{\multicolumn{3}{|c|}{$\begin{array}{l}\mathrm{PD}^{* *} \\
\mathrm{dia}^{-1}\end{array}$}} & \multirow{2}{*}{\multicolumn{3}{|c|}{$\frac{P V L^{* *}}{\text { vaca }^{-1} \mathrm{dia}^{-1}}$}} & \multirow{2}{*}{\multicolumn{3}{|c|}{$\frac{\mathrm{RVL} / \mathrm{VT}^{* *}}{\%}$}} & \multirow{2}{*}{\multicolumn{3}{|c|}{$\frac{\mathrm{PMO}^{* *}}{\mathrm{~L} \mathrm{dia}^{-1} \text { home }^{-1}}$}} & \multirow{2}{*}{\multicolumn{3}{|c|}{$\frac{\mathrm{PT}^{\star \star}}{\mathrm{L} \mathrm{ha}^{-1} \text { ano }^{-1}}$}} & \multirow{2}{*}{\multicolumn{3}{|c|}{$\frac{C L L^{* *}}{\mathrm{~kg} \mathrm{~L}^{-1} \text { leite } e^{-1}}$}} \\
\hline & & & & & & & & & & & & & & & & & & \\
\hline & 563 & \pm & 59 & 15,7 & \pm & 1,2 & 72,5 & \pm & 3,1 & 104 & \pm & 16,0 & 2.039 & \pm & 221 & 0,30 & \pm & 0,04 \\
\hline 2 & 537 & \pm & 29 & 15,9 & \pm & 3,5 & 70,9 & \pm & 4,2 & 101 & \pm & 8,6 & 1.759 & \pm & 89 & 0,27 & \pm & 0,02 \\
\hline 3 & 371 & \pm & 31 & 9,9 & \pm & 0,2 & 63,4 & \pm & 3,5 & 167 & \pm & 17,1 & 734 & \pm & 133 & 0,40 & \pm & 0,08 \\
\hline 4 & 348 & \pm & 21 & 15,8 & \pm & 1,6 & 74,1 & \pm & 1,3 & 105 & \pm & 12,6 & 7.075 & \pm & 357 & 0,25 & \pm & 0,03 \\
\hline 5 & 268 & \pm & 3 & 12,7 & \pm & 0,7 & 66,0 & \pm & 1,7 & 103 & \pm & 1,6 & 1.180 & \pm & 14 & 0,27 & \pm & 0,01 \\
\hline 6 & 267 & \pm & 28 & 13,6 & \pm & 1,4 & 76,1 & \pm & 3,6 & 100 & \pm & 23,1 & 3.600 & \pm & 421 & 0,25 & \pm & 0,06 \\
\hline 7 & 213 & \pm & 10 & 15,9 & \pm & 0,5 & 79,1 & \pm & 1,9 & 126 & \pm & 18,6 & 2.159 & \pm & 108 & 0,30 & \pm & 0,03 \\
\hline 8 & 192 & \pm & 21 & 12,3 & \pm & 0,4 & 87,5 & \pm & 2,3 & 78 & \pm & 4,3 & 2.310 & \pm & 220 & 0,43 & \pm & 0,03 \\
\hline 9 & 158 & \pm & 13 & 12,6 & \pm & 1,0 & 74,4 & \pm & 2,1 & 83 & \pm & 2,2 & 2.806 & \pm & 533 & 0,22 & \pm & 0,03 \\
\hline 10 & 144 & \pm & 20 & 5,3 & \pm & 0,3 & 64,5 & \pm & 5,0 & 67 & \pm & 3,8 & 463 & \pm & 70 & 0,25 & \pm & 0,04 \\
\hline 11 & 103 & \pm & 2 & 7,8 & \pm & 1,9 & 73,3 & \pm & 2,3 & 71 & \pm & 17,3 & 629 & \pm & 154 & 0,35 & \pm & 0,04 \\
\hline 12 & 103 & \pm & 3 & 12,5 & \pm & 0,4 & 82,5 & \pm & 1,5 & 77 & \pm & 10,6 & 678 & \pm & 16 & 0,25 & \pm & 0,04 \\
\hline 13 & 95 & \pm & 21 & 5,8 & \pm & 0,1 & 68,4 & \pm & 2,8 & 58 & \pm & 4,7 & 728 & \pm & 31 & 0,19 & \pm & 0,05 \\
\hline 14 & 91 & \pm & 7 & 8,4 & \pm & 0,9 & 69,4 & \pm & 4,5 & 52 & \pm & 10,4 & 1.100 & \pm & 90 & 0,38 & \pm & 0,04 \\
\hline 15 & 87 & \pm & 11 & 6,6 & \pm & 0,7 & 58,4 & \pm & 0,8 & 82 & \pm & 14,5 & 2.683 & \pm & 327 & 0,27 & \pm & 0,08 \\
\hline 16 & 84 & \pm & 3 & 8,9 & \pm & 0,2 & 73,8 & \pm & 1,9 & 34 & \pm & 2,5 & 1.663 & \pm & 70 & 0,31 & \pm & 0,01 \\
\hline 17 & 84 & \pm & 11 & 8,8 & \pm & 1,0 & 67,9 & \pm & 4,9 & 82 & \pm & 11,2 & 811 & \pm & 12 & 0,38 & \pm & 0,06 \\
\hline 18 & 65 & \pm & 11 & 9,7 & \pm & 0,4 & 84,3 & \pm & 3,9 & 43 & \pm & 10,1 & 1.212 & \pm & 175 & 0,32 & \pm & 0,02 \\
\hline 19 & 60 & \pm & 5 & 9,4 & \pm & 0,5 & 74,7 & \pm & 5,1 & 50 & \pm & 6,9 & 637 & \pm & 47 & 0,30 & \pm & 0,03 \\
\hline 20 & 47 & \pm & 23 & 4,7 & \pm & 1,0 & 53,1 & \pm & 11,2 & 25 & \pm & 14,2 & 369 & \pm & 141 & 0,27 & \pm & 0,08 \\
\hline 21 & 46 & \pm & 8 & 8,5 & \pm & 1,0 & 61,6 & \pm & 8,0 & 23 & \pm & 5,0 & 923 & \pm & 167 & 0,41 & \pm & 0,03 \\
\hline 22 & 27 & \pm & 6 & 4,4 & \pm & 1,0 & 58,0 & \pm & 7,6 & 30 & \pm & 6,9 & 1.014 & \pm & 207 & 0,48 & \pm & 0,11 \\
\hline
\end{tabular}

*Média e desvio-padrão da média $(m \pm d p)$.

${ }^{* *} \mathrm{PR}=$ Propriedades; $\mathrm{PD}=$ Produção diária; $\mathrm{PVL}=$ Produção por vaca em lactação; $R V L / T V$ = Relação de vacas em lactação pelo total de vacas no rebanho; $\mathrm{PMO}=$ Produtividade da mão-de-obra; $\mathrm{PT}=$ Produtividade da terra; $\mathrm{CLL}=$ Concentrado por litro de leite produzido. 
intervalo entre partos, alimentação, sanidade e característica racial contribuíram para este baixo desempenho.

Nesse trabalho, foi feita uma simulação da produtividade de um rebanho com 50 cabeças, de mesmo potencial genético, durante dois anos, e com diferentes intervalos entre partos. Maiores detalhes sobre essa simulação são apresentados em Oliveira (1999). Com base nas citações de Faria \& Silva (1996), considerou-se o decréscimo de produção de $7 \%$ ao mês, após atingido o pico de lactação, entre aproximadamente 50 e 70 dias de lactação. Reduzindo-se o intervalo entre partos (IP) de 15 para 12 meses, há uma elevação de aproximadamente $15 \%$ na produção de leite (Figura 1). Assim, pode-se verificar que aumentos de produtividade são possíveis apenas reduzindo-se o intervalo entre partos.

Os índices de produtividade da terra (PT) e da mão-de-obra (PMO) encontram-se na TABELA 2. A produtividade por vaca em lactação ( $P V L)$ e consequentemente a produtividade pelo total de vacas no rebanho exercem influência na produtividade da terra, pois a propriedade número 4 (TABELA 2), que tem alta PVL, teve a maior PT, $7.075 \mathrm{~L} \mathrm{ha}^{-1} \mathrm{ano}^{-1}$, enquanto a propriedade número 20 , com baixa $\mathrm{PVL}$, apresentou a menor PT, $367 \mathrm{~L} \mathrm{ha}^{-1} \mathrm{ano}^{-1}$, correspondente a aproximadamente $\mathrm{L} \mathrm{ha}^{-1} \mathrm{dia}^{-1}$.

Em Minas Gerais, levantamento realizado pela Federação da Agricultura do Estado de Minas Gerais (1996) mostrou que os índices de produtividade da terra na Região da Zona da Mata mineira têm variado de 506 a $1.300 \mathrm{~L} \mathrm{ha}^{-1} \mathrm{ano}^{-1}$. No vale do Paraíba (SP), Noronha et al. (1990) obtiveram índices de PT que variaram de 833 a $2.681 \mathrm{~L} \mathrm{ha}^{-1} \mathrm{ano}^{-1}$, entretanto, para o Médio
Paraíba (RJ), Mondani (1996) verificou índices menores, situando-se entre 580 e 680, e em Valença (RJ), Teixeira \& Calegar (1998) encontraram produtividade oscilando de 1.800 a $3.600 \mathrm{~L} \mathrm{ha}^{-1}$ ano $^{-1}$.

No Estado de São Paulo, fazendas que se destacaram pelos seus índices de produtividade apresentaram índices de PT variando de 7.500 a 9.700 $\mathrm{L} \mathrm{ha}^{-1}$ ano $^{-1}$ (Gomes, 1997). Portanto, considerando uma taxa de lotação de 1,0 vaca por ha (Mondani, 1996) e tendo-se rebanho com $70 \%$ de vacas em lactação, apresentando produtividade média de $6 \mathrm{~L} \mathrm{dia}^{-1}$, verificase que produtividades da terra próximas a 1.500 $\mathrm{L} \mathrm{ha}^{-1} \mathrm{ano}^{-1}$ podem ser facilmente obtidas.

A produtividade da mão-de-obra (TABELA 2) variou de 23 a 167 litros por dia por homem $\left(\mathrm{L} \mathrm{d}^{-1} \mathrm{~h}^{-1}\right)$, sendo a média dessas 22 propriedades $76 \mathrm{~L} \mathrm{~d}^{-1} \mathrm{~h}^{-1}$. No levantamento realizado pela Federação da Agricultura do Estado de Minas Gerais (1996), a PMO oscilou de 28 a $83 \mathrm{~L} \mathrm{~d}^{-1} \mathrm{~h}^{-1} \mathrm{e}$, nestas propriedades, maior produção diária levou a maior produtividade da mão-de-obra.

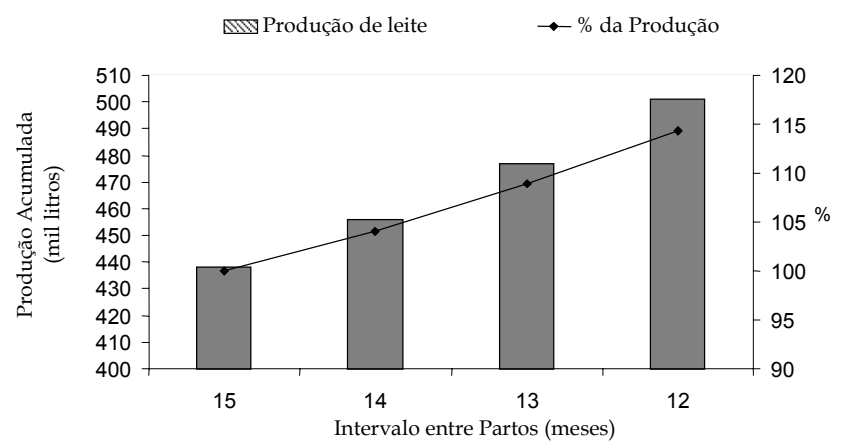

Figura 1 - Aumento de produção de leite resultante de diferentes intervalos entre partos.

TABELA 3 - Indicadores técnicos das propriedades leiteiras, no período de maio de 1996 a abril de 1998, que apresentaram resultados negativos para $M L$ e TRI considerando a terra.

\begin{tabular}{|c|c|c|c|c|c|c|c|c|c|c|c|c|}
\hline \multirow{2}{*}{\multicolumn{2}{|c|}{ Índice técnico* }} & \multicolumn{11}{|c|}{ Propriedade } \\
\hline & & \multirow[t]{2}{*}{8} & \multirow[t]{2}{*}{12} & \multirow{2}{*}{$\begin{array}{l}14 \\
\underline{91}\end{array}$} & \multirow{2}{*}{$\begin{array}{l}15 \\
87 \\
\end{array}$} & \multirow{2}{*}{$\begin{array}{l}16 \\
\underline{84}\end{array}$} & \multirow{2}{*}{$\begin{array}{l}17 \\
\underline{84}\end{array}$} & \multirow{2}{*}{$\begin{array}{l}18 \\
\underline{65} \\
\end{array}$} & \multirow{2}{*}{$\begin{array}{l}19 \\
\underline{63} \\
\end{array}$} & \multirow{2}{*}{$\begin{array}{l}20 \\
\underline{62}\end{array}$} & \multirow{2}{*}{$\begin{array}{l}21 \\
\underline{46}\end{array}$} & \multirow{2}{*}{$\begin{array}{l}22 \\
\underline{27} \\
\end{array}$} \\
\hline חק & $<100 \mathrm{~L}$ & & & & & & & & & & & \\
\hline 50 & $>100 \mathrm{~L}$ & 169 & 103 & & & & & & & & & \\
\hline \multirow{2}{*}{ PVL } & $<10 \mathrm{~L}$ & & & $\underline{8,4}$ & $\underline{6,6}$ & $\underline{8,9}$ & $\underline{8,8}$ & $\underline{9,7}$ & $\underline{9,4}$ & $\underline{4,7}$ & $\underline{8,5}$ & $\underline{4,4}$ \\
\hline & $>10 \mathrm{~L}$ & 12,3 & 12,5 & & & & & & & & & \\
\hline \multirow{2}{*}{ RVL/ TV } & $<70 \%$ & & & $\underline{69}$ & $\underline{58}$ & & $\underline{68}$ & & & $\underline{53}$ & $\underline{61}$ & $\underline{58}$ \\
\hline & $>70 \%$ & 87 & 82 & & & 73 & & 84 & 74 & & & \\
\hline \multirow{2}{*}{ PMO } & $<100 \mathrm{~L}$ & $\underline{78}$ & $\underline{77}$ & $\underline{52}$ & $\underline{82}$ & $\underline{34}$ & $\underline{82}$ & $\underline{43}$ & $\underline{50}$ & $\underline{25}$ & $\underline{23}$ & $\underline{30}$ \\
\hline & $>100 \mathrm{~L}$ & & & & & & & & & & & \\
\hline \multirow{2}{*}{ PT } & $<1.000 \mathrm{~L}$ & & $\underline{678}$ & & & & $\underline{811}$ & & $\underline{637}$ & $\underline{367}$ & $\underline{923}$ & \\
\hline & $>1.000 \mathrm{~L}$ & 2.310 & & 1.100 & 2.683 & 1.663 & & 1.212 & & & & 1.014 \\
\hline \multirow{2}{*}{ CLL } & $<300 \mathrm{~g}$ & & 248 & & 280 & & & & & 265 & & \\
\hline & $>300 \mathrm{~g}$ & $\underline{427}$ & & $\underline{382}$ & & $\underline{314}$ & $\underline{380}$ & $\underline{321}$ & $\underline{303}$ & & $\underline{412}$ & $\underline{478}$ \\
\hline
\end{tabular}

*Os índices técnicos sublinhados e em negrito estão fora dos limites técnicos estipulados. 
O consumo de concentrado por litro de leite produzido é mostrado na TABELA 2, onde pode-se observar que o índice, $\mathrm{kg}$ de concentrado por litro de leite, variou de 0,190 a 0,480 . Os valores obtidos por Gomes (1997) em três fazendas do Estado de São Paulo, que se destacaram pelos seus índices de produtividade, foram $0,320 \mathrm{~kg}, 0,330 \mathrm{~kg}$ e $0,290 \mathrm{~kg}$ de concentrado por litro de leite. Mondani (1996), em estudo conduzido na Região do Médio Paraíba do Estado do Rio de Janeiro, verificou que o consumo de concentrado oscilou de 0,340 a 0,390 kg por litro de leite.

Uma vez que a melhoria destes índices técnicos pode ser alcançada por diferentes combinações dos fatores de produção, sugerem-se investimentos em corretivos de acidez do solo, fertilizante e inseminação artificial. Constatou-se por intermédio das visitas técnicas que aproximadamente $80 \%$ das propriedades têm investido na melhoria do padrão racial, conseguida pela inseminação artificial e na qualidade do leite, através de ordenha mecânica e resfriamento. A correta exploração desses fatores de produção poderá melhorar significativamente os índices técnicos destas propriedades e, conseqüentemente, os índices econômicos.

\section{CONCLUSÕES}

O aumento da produtividade animal foi a alternativa mais viável para o aumento da rentabilidade e, várias propriedades podem elevar a produtividade animal apenas combatendo as causas que impedem menor intervalo entre partos.

Investimentos em corretivos de acidez do solo, fertilizantes, inseminação artificial e qualidade do leite através de ordenha mecanizada e resfriamento do produto logo após a ordenha certamente contribuirão para a melhoria dos resultados econômicos.

\section{AGRADECIMENTOS}

À CAPES/PICDT e a Universidade Federal de Viçosa pelo apoio financeiro na condução deste trabalho.

\section{REFERÊNCIAS BIBLIOGRÁFICAS}

EMPRESA BRASILEIRA DE PESQUISA AGROPECUÁRIA. Centro Nacional de Pesquisa de Gado de Leite. Relatório do projeto de acompanhamento de fazendas como instrumento para análise de sistemas de produção de leite (período 1986-1990). Coronel Pacheco: EMBRAPA, CNPGL, 1991.
FEDERAÇÃO DA AGRICULTURA DO ESTADO DE MINAS GERAIS. Diagnóstico da pecuária leiteira do estado de Minas Gerais: relatório de pesquisa. Belo Horizonte: FAEMG; SEBRAE-MG, 1996. 102p.

FEDERAÇÃO DA AGRICULTURA DO ESTADO DO PARANÁ. Sistema de acompanhamento do custo de produção do leite no Paraná. Curitiba: FAEP; OCEPAR, 1996. 99p.

FARIA, V.P. Pecuária leiteira no mundo e no Brasil. In: FARIA, V.P. (Coord.) Produção de leite: conceitos básicos. Piracicaba: FEALQ, 1988. v.3, p.5-12.

FARIA, V.P.; SILVA, S.C. Fatores biológicos determinantes de mudanças na pecuária leiteira. In: SIMPÓsIO INTERNACIONAL O FUTURO DOS SISTEMAS DE PRODUÇÃO DE LEITE NO BRASIL, Juiz de Fora, 1996. Anais. Juiz de Fora, 1996. p.77-89.

GOMES, A.T.; CASTRO, F.G.; ASSIS, A.G. Análise técnicoeconômica de sistemas de produção de leite. Coronel Pacheco: EMBRAPA, CNPGL, 1986. 34p. (Documentos, 30).

GOMES, S.T. Indicadores de eficiência técnica e econômica na produção de leite. São Paulo: FAESP, 1997. 178p.

MARTINS, P.C. Análise comparativa entre o sistema de produção de leite da EMBRAPA e sistemas de produção em fazendas do Estado de Minas Gerais. Viçosa, 1988. 108p. Dissertação (M.S.) - Universidade Federal de Viçosa.

MATARAZZO, D.C. Análise financeira de balanços: abordagem básica. 2.ed. São Paulo: Atlas, 1992. cap.4, p.95-133.

MELO FILHO, G.A.; RICHETTI, A. Manual de contas da empresa rural: orientações gerais. Dourados: EMBRAPA CPAO, 1998. 61p. (Documentos, 17).

MONDANI, I. A rentabilidade da atividade leiteira: um caso de produtores no Médio Paraíba do Estado do Rio de Janeiro. Lavras, 1996. 83p. Dissertação (Mestrado) - Universidade Federal de Lavras.

NORONHA, J.F.; ALCANTARA, J.M.; PETRI, M.A.A. Custos de produção e análise econômica da atividade leiteira. In: SIMPÓSIO SOBRE PRODUÇÃO ANIMAL, 7., Campinas, 1990. Anais. Piracicaba: FEALQ, 1990. p.109-140.

OLIVEIRA, T.B.A.O. Análise das eficiências técnicas e econômicas em propriedades assistidas pelo Programa de Desenvolvimento da Pecuária Leiteira da Região de Viçosa, Minas Gerais. São Carlos, 1999. 99p. Dissertação (Mestrado) - Universidade Federal de São Carlos.

SCHIFFLER, E.A. Análise de eficiência técnica e econômica de sistemas de produção de leite na Região de São Carlos, São Paulo. Viçosa, 1998. 128p. Dissertação (M.S.) - Universidade Federal de Viçosa.

TEIXEIRA, S.R.; CALEGAR, G.M. Fazenda Santa Tereza: o caso de um produtor de leite em transição. In: REUNIÃO DA SOCIEDADE BRASILEIRA DE ZOOTECNIA, 35., Botucatu, 1998. Anais. Botucatu: SBZ, 1998. p.105-107.

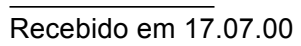

